\title{
Importance of n-3 PUFA consumption during pregnancy: perception discrepancies between pregnant women and gynaecologists-obstetricians in Belgium
}

\author{
Axelle Hoge ${ }^{1, *}$, Florence Bernardy ${ }^{1}$, Anne-Françoise Donneau ${ }^{1}, N^{\prime}$ dia Dardenne ${ }^{1}$, \\ Sylvie Degée 2 , Michelle Nisolle ${ }^{2}$, Michèle Guillaume ${ }^{1, \dagger}$ and Vincenzo Castronovo ${ }^{3,} \dagger$ \\ ${ }^{1}$ Department of Public Health, University of Liège, Avenue Hippocrate 13- B23, 4000 Liège, Belgium: ${ }^{2}$ Department \\ of Obstetrics and Gynecology, CHR Citadelle Hospital, University of Liège, Liège, Belgium: ${ }^{3}$ Metastasis Research \\ Laboratory, GIGA-CANCER, University of Liège, Liège, Belgium
}

Submitted 8 June 2018: Final revision received 19 November 2018: Accepted 3 January 2019: First published online 20 February 2019

\begin{abstract}
Objective: $n$-3 PUFA during pregnancy have been subject to intense research interest, but also much controversy, creating a situation of confusion among patients and health-care providers. The present study was carried out to explore knowledge, attitude and practices regarding n-3 PUFA in two independent populations of pregnant women and gynaecologists-obstetricians in Belgium and to assess the relationship between the pregnant women's attributes and their $n-3$ PUFA status measured by the omega-3 index.

Design: Cross-sectional study. Knowledge, attitude and practices were collected by self-administered questionnaires while laboratory data were available for each pregnant woman.

Setting: During the first antenatal hospital appointment (for the pregnant women) and by email (for the gynaecologists-obstetricians).

Participants: Women in early pregnancy ( $n$ 122) and gynaecologists-obstetricians ( $n$ 67).

Results: Marked discrepancies in perception were evidenced between the pregnant women and health-care providers. While $82 \%$ of the women attached high importance to $n-3$ PUFA during pregnancy, only a third of the gynaecologists-obstetricians did. About $35 \%$ of the women declared paying particular attention to their consumption of $n-3$ PUFA. After adjusting for sociodemographic characteristics, these positive dietary practices were significantly associated with higher omega-3 index $(P=0.04)$. Overall, $43.3 \%$ of professionals did not provide any information about $n$-3 PUFA to their pregnant patients and $46 \cdot 3 \%$ did not take any preventive actions.

Conclusions: Evidence-based guidelines, refreshment training and communication tools are needed to improve awareness and clinical practices among caregivers regarding $n$-3 PUFA to benefit both mothers and their children.
\end{abstract}

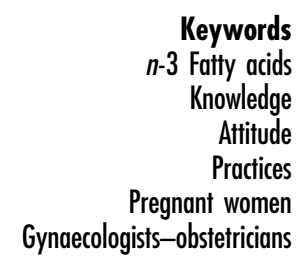

Keywords

3 Fatty acids

Altedge

Practices

Gynaecologists-obstetricians
Research has clearly established a link between $n$-3 PUFA and general health ${ }^{(1,2)}$. Their longer-chain derivatives $(n-3$ LCPUFA) are significant structural components of cell membrane phospholipids throughout the body and are especially rich in the central nervous system ${ }^{(3)}$. The large accretion of DHA observed in the fetal brain and retina during the second half of gestation is required to promote their optimal growth and development ${ }^{(4,5)}$. More recently, several studies have shown that DHA supply in early life is

\footnotetext{
† Michèle Guillaume and Vincenzo Castronovo are joint senior authors.
}

a major determinant of visual and neurocognitive functions in the offspring ${ }^{(6,7)}$.

Additional evidence supports the hypothesis that $n-3$ PUFA positively affect numerous maternal and child health outcomes. Lower incidence of low birth weight and preterm birth $^{(8)}$, reduced risk of pre-eclampsia ${ }^{(9)}$ and perinatal depression $^{(10)}$ as well as the primary prevention of asthma and allergies in early childhood and childhood ${ }^{(11)}$ have been associated with $n$-3 LCPUFA.

Since neither the placenta nor the fetus possesses sufficient desaturase activity to synthesize long-chain PUFA, 
the primary source of these fatty acids for the fetus comes from maternal stores and dietary intake ${ }^{(12)}$. Guidelines advise pregnant women to consume 250-500 mg of EPA plus DHA daily, including at least $200 \mathrm{mg}$ of DHA daily ${ }^{(13,14)}$. The European Food Safety Authority recommends an additional intake of 100-200 mg of DHA daily ${ }^{(15)}$. However, the Western diet is relatively deficient in $n-3$ PUFA. Food surveys have also reported insufficient dietary intakes of $n-3$ LCPUFA among a large portion of European pregnant women ${ }^{(16-19)}$. The Belgian food-based dietary guidelines appear to specifically encourage the general population to consume dietary sources of $n-3$ PUFA, including two portions of fish per week of which at least one portion is oily fish ${ }^{(20)}$. Longitudinal studies focusing on biochemical fatty acid markers indicate that pregnancy is associated with a significant decrease in maternal DHA status $^{(21-23)}$. These observations suggest that under present dietary conditions, mothers may be unable to meet these needs due to the high fetal DHA requirements.

Although seafood is the main dietary source of $n-3$ LCPUFA, it may also be contaminated by methylmercury, a neurotoxin that is particularly harmful to the developing fetal brain ${ }^{(24)}$. Warning messages about the adverse health impact of mercury exposure may have led to women dramatically reducing their fish consumption ${ }^{(25-27)}$. A survey in Belgium highlighted that consumers' awareness of the potential toxicity of fish consumption is higher than their awareness of the potential health benefits ${ }^{(28)}$.

Several nutrients are of utmost importance during pregnancy and need to be discussed during antenatal care. Pregnant women reported receiving much less information regarding the importance and role of $n-3$ PUFA during pregnancy than regarding other nutrients such as folate, iron and calcium ${ }^{(29)}$. Health-care providers are a trusted source of pregnancy-related dietary information ${ }^{(30)}$ and can influence the eating practices of their pregnant patients ${ }^{(31)}$. According to the study of Bloomingdale et al., pregnant women might indeed be willing to increase their fish consumption if they received appropriate advice from their obstetrician ${ }^{(32)}$.

Although some studies have focused on women's awareness of the importance of $n$ - 3 LCPUFA or fish consumption during pregnancy ${ }^{(29,32,33)}$, there have been no studies investigating this topic among front-line healthcare professionals. Therefore, the aims of the present study were to: (i) provide an insight into the knowledge, attitude and practices of pregnant women and gynaecologists-obstetricians regarding $n$-3 PUFA; and (ii) study the relationship between knowledge, attitude and dietary practices regarding $n-3$ PUFA of pregnant women and their $n$ - 3 PUFA status measured by the omega- 3 index.

\section{Materials and methods}

\section{Study design and populations}

The recruitment of the pregnant women took place between February and August 2016 in the Obstetrics and
Gynecology Department of the CHR Liège Hospital, Liège, Belgium. The women were invited to complete a selfadministered questionnaire while waiting for their first antenatal appointment. Inclusion criteria were: (i) being between the 7 th and 18th week of gestation; (ii) being free from any chronic diseases such as hypertension and diabetes; and (iii) presenting with a singleton pregnancy. All women were informed about the purpose of the study and written consent was obtained prior to completing the survey.

The second study population consisted of all gynaecologists-obstetricians with an email address $(n$ 452) from the Belgian Group of French Language GynecologistsObstetricians (GGOLFB). They were invited to complete an anonymous online survey via email between February and May 2016. The investigators have not been able to link email address and sociodemographic data. The completion of the survey indicated the consent from gynaecologists-obstetricians to participate in the study.

The pregnant women and the gynaecologists-obstetricians were recruited independently.

The study protocol was reviewed and approved by the Ethics Committee of the CHR Liège Hospital, Liège, Belgium (B412201526650).

\section{Study instruments}

The pregnant women completed a self-administered questionnaire on sociodemographic characteristics as well as knowledge, attitude and dietary practices regarding n-3 PUFA during pregnancy. Maternal characteristics included age, parity, nationality and level of education. Level of education was expressed as low (primary and lower and upper secondary) and high (non-university degree and university degree). General beliefs about $n$-3 PUFA and sources of received information were investigated at the beginning of the survey by two open-ended questions. Specifically, one question on pregnant women's beliefs was: 'What did you know about omega-3 fatty acids?' Pregnant women who cited one or several correct facts about $n$-3 PUFA without prompting were classified as having good knowledge. After a brief definition of 'omega-3 fatty acids', closed-ended questions were used to assess participants' knowledge about the health benefits of $n$-3 PUFA during pregnancy and dietary sources of $n-3$ PUFA. Simply stating that 'These are good fats, most of which must be provided by the diet' allowed participants to make a connection with their prior knowledge. As a result, more people were able to answer to the closed-ended questions about health benefits and dietary sources of $n$-3 PUFA. Attitude was assessed by the question: 'What importance do you attach to omega-3 fatty acids during pregnancy?' The answers were classified as 'moderate or high importance' and 'no or little importance'. One question also explored what participants considered would be the best source of information about 
$n-3$ PUFA during pregnancy. Finally, the pregnant women were invited to communicate any dietary changes made since pregnancy, with a focus on fats. Specifically, the questions were: 'Since pregnancy, have you modified your diet? - If yes, how?' and 'Since pregnancy, have you paid particular attention to your fat intake? - If yes, how?' Detailed information on use of $n$ - 3 PUFA supplements was also collected, including product name, type of medication, dose, frequency and duration of use.

The online survey created for gynaecologists-obstetricians included questions about demographic characteristics as well as knowledge, attitude and clinical practices regarding $n$-3 PUFA during pregnancy. Knowledge was assessed by questions asking about the frequency of low $n-3$ PUFA status and existing guidelines on $n-3$ PUFA. For example, the first question was: 'In your opinion, pregnant women have suboptimal omega-3 polyunsaturated fatty acid levels in blood: never, sometimes, often or always?' Suboptimal $n$ - 3 PUFA status was then described as 'nonexistent or occasional' ('never' or 'sometimes') or 'common' ('often' and 'always'). The questionnaire also explored the need and the desire for further education on the topic. Attitude was assessed using the same question as for the pregnant women. Concerning nutritional care practices, questions were related to the monitoring of $n-3$ PUFA status in pregnant women, the implementation of preventive actions in relation to a possible suboptimal $n-3$ PUFA status, and the transmission of information on the importance and/or the role of $n$ - 3 PUFA during pregnancy. The opinion of gynaecologists-obstetricians on routine $n$-3 LCPUFA supplementation was also researched. The question was: 'Do you think that the provision of systematic $n$-3 LCPUFA supplementation to pregnant women would be necessary? - For what reasons?'

\section{Erythrocyte n-3 PUFA analysis}

Fasting blood specimens were collected from each pregnant woman at recruitment using an EDTA tube for testing for omega-3 index. Erythrocyte fatty acid composition was analysed by GC as previously described ${ }^{(34)}$. The omega-3 index (IOM3) was defined as erythrocyte EPA plus DHA expressed as weight percentage of total fatty acids. It reflects the long-term intake of $\mathrm{EPA}$ and $\mathrm{DHA}^{(35)}$. In cardiology, IOM3 was proposed as a marker, and even as a risk factor, for cardiovascular events (e.g. sudden cardiac death). IOM3 $\geq 8 \%$ was suggested as optimal for reducing risk $^{(36,37)}$.

\section{Statistical analysis}

Open-ended questions were coded before analysis. Classical descriptive statistics were used to describe the general characteristics of the study participants. For quantitative variables, results were expressed as mean and SD. Frequency tables were used to summarize categorical variables. The $\chi^{2}$ test was applied to compare proportions of categorical variables between groups. Multiple regression analysis was used to test the effect of the pregnant women's dietary practices on omega- 3 index. A logarithmic transformation was applied to omega- 3 index values to normalize their distribution. The statistical analysis also investigated the direct, indirect and total effects of knowledge and attitude on omega-3 index in the pregnant women, with dietary practices as mediating variable (Fig. 1). The mediation effect was tested using the method described by Valeri and Vanderweele ${ }^{(38)}$, which allows dichotomous mediators. The bootstrapping method was used to test for the significance of the direct, indirect and total effect using 95\% CI. A statistically significant indirect effect implies the presence of mediation. There is 'partial mediation' when the direct effect is significantly different from 0 and 'complete mediation' if it is not. Both regression and mediation models were adjusted for the pregnant women's characteristics (age, parity, nationality and level of education). $P<0.05$ was considered statistically significant. Statistical analysis was performed using the statistical software package SAS version 9.4 and mediation analysis was performed by using the macro\% mediation $^{(38)}$.

\section{Results}

\section{Characteristics of populations}

A total of 122 pregnant women participated in the study. Their characteristics are summarized in Table 1 . The women were 28.3 (SD 5.6) years old and $62.3 \%$ had a low educational level. There were sixty-eight ( $55.7 \%$ ) Belgian Caucasians, nineteen $(15.6 \%)$ came from North Africa or the Middle East, nine $(7.4 \%)$ from Central Africa, eight (6.6\%) from Asia, seven (5.7\%) from West Africa, seven (5.7\%) from South Europe, three (2.5\%) from West Europe and one (0.8\%) from East Africa. Fifty-one ( $41.8 \%)$ participants were nulliparous (no previous birth). The median (interquartile range) omega-3 index was 5.51 (4.73-6.67)\%. A majority of participants (91.8\%) exhibited a low $n$-3 LCPUFA status (IOM3 < $\%$ ).

Sixty-seven gynaecologists-obstetricians (15\% participation rate) completed the survey. Their characteristics are

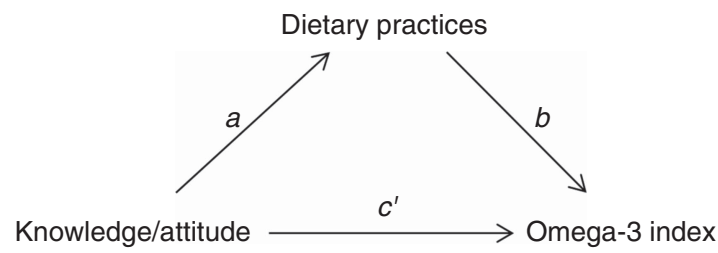

Fig. 1 Path diagram of simple mediation, investigating dietary practices as a mediator between knowledge and attitude and omega-3 index ( $a$, knowledge/attitude $v$. dietary practices relationship; $b$, dietary practices $v$. omega-3 index relationship, controlling for knowledge/attitude; $c^{\prime}$, direct effect; function of $a$ and $b$, indirect effect; total effect $=$ direct + indirect effects) 
Table 1 Sociodemographic characteristics of pregnant women ( $n$ 122) sampled in CHR Liège Hospital, Liège, Belgium, FebruaryAugust 2016

\begin{tabular}{lcc}
\hline & \multicolumn{2}{c}{ Summary statistics } \\
\cline { 2 - 3 } Variable & Mean or $n$ & SD or \% \\
\hline Age (years), mean and SD & 28.3 & $5 \cdot 6$ \\
Parity, $n$ and \% & 51 & 41.8 \\
$\quad$ Nulliparous & 41 & 33.6 \\
Primiparous & 28 & 23.0 \\
Multiparous & 2 & $1 \cdot 64$ \\
$\quad$ Not know & & \\
Nationality, $n$ and \% & 68 & 55.7 \\
$\quad$ Belgian & 54 & 44.3 \\
Other & & \\
Level of education, $n$ and \% & 76 & 62.3 \\
$\quad$ Low & 46 & 37.7 \\
High & & \\
\hline
\end{tabular}

displayed in Table 2 . Thirty (44.8\%) professionals worked in a hospital and twenty-four (35.8\%) had multiple workplaces (hospital, private home and/or health-care centre). Almost two-thirds of participants (64.2\%) had a professional experience of over 10 years.

\section{Knowledge, attitude and dietary practices of the pregnant women}

Among respondents, seventy-six (62.3\%) had already heard of or read something about $n-3$ PUFA of whom forty-six (60.5\%) had good knowledge about $n$-3 PUFA. The answers to these open-ended questions are summarized in Table 3. A quarter of the women correctly reported that $n$ - 3 PUFA are essential fats because the body cannot synthesize them and that they must be obtained from foods. The same proportion recognized that $n-3$ PUFA are beneficial nutrients for health, particularly for the heart, the nervous system or the brain. Approximately $20 \%$ of the women accurately cited fish/oily fish and fish oil as dietary sources and $17 \cdot 3 \%$ correctly emphasized that $n$-3 PUFA positively affect maternal and child health

Table 2 Demographic characteristics of gynaecologists-obstetricians (n 67) sampled from the Belgian Group of French Language Gynecologists-Obstetricians, February-May 2016

\begin{tabular}{lrc}
\hline & \multicolumn{2}{c}{$\begin{array}{c}\text { Summary } \\
\text { statistics }\end{array}$} \\
\cline { 2 - 4 } Variable & $n$ & $\%$ \\
\hline Sex & & \\
$\quad$ Men & 26 & $38 \cdot 8$ \\
$\quad$ Women & 41 & $61 \cdot 2$ \\
Workplace & 30 & 44.8 \\
$\quad$ Hospital only & 24 & $35 \cdot 8$ \\
Multiple (hospital, private home and/or health-care & & \\
$\quad$ centre) & 12 & 17.9 \\
Private practice only & 1 & 1.49 \\
$\quad$ Health-care centre only & & \\
Professional experience & 24 & 35.8 \\
$\quad<10$ years & 43 & 64.2 \\
$\quad \geq 10$ years & &
\end{tabular}

Table 3 Pregnant women's answers to the question 'What did you know about omega-3 fatty acids?' $(n$ 122) and sources where pregnant women get their information ( $n 76)$, Belgium, FebruaryAugust 2016

\begin{tabular}{lr}
\hline Variable & $\%^{*}$ \\
\hline Pregnant women's beliefs about $n$-3 PUFA & \\
Classified as right answers & $25 \cdot 3$ \\
Are essential fatty acids & $25 \cdot 3$ \\
Are beneficial nutrients for the health, particularly for the & \\
$\quad$ heart, the nervous system or the brain & $18 \cdot 7$ \\
Are derived from fish/oily fish or fish oil supplements & $17 \cdot 3$ \\
Positively affect maternal and child health outcomes & $2 \cdot 7$ \\
Affect membrane fluidity & \\
Classified as wrong answers & $8 \cdot 0$ \\
Are involved in muscle regeneration, iron status, ... & $2 \cdot 7$ \\
Are antioxidant vitamins & $16 \cdot 0$ \\
Nothing & \\
Sources of information & $56 \cdot 0$ \\
Media, television, Internet, books or magazines & $37 \cdot 3$ \\
Health-care providers & $26 \cdot 7$ \\
School & $12 \cdot 0$ \\
Family or friends & 4.0 \\
Not remembered &
\end{tabular}

*Percentages do not add up to $100 \%$, because multiple answers were accepted.

outcomes. Among wrong beliefs, the women claimed that $n$-3 PUFA acids are antioxidant vitamins or that they are involved in muscle regeneration. Information was primarily derived from the media, Internet, books or magazines $(56.0 \%)$, from health-care providers $(37.3 \%)$ or school (26.7\%). Among the women who cited health-care providers as source of information, information was given by the sole gynaecologist-obstetrician for sixteen out of twenty-eight $(57 \cdot 1 \%)$ women, by the gynaecologistobstetrician plus other professionals for five (17.9\%) women, by the midwife for one (3.6\%) woman and by other professionals not related to the pregnancy for six (21.4\%) women. The women who received information from their gynaecologist-obstetrician were significantly more likely to demonstrate knowledge related to health benefits of $n$-3 PUFA during pregnancy than the uninformed women $(33.3 v$ v. 5.6\%; $P=0 \cdot 0015)$.

From the semi-closed questions, sixty-nine (56.6\%) participants replied that they did not know the health benefits of $n$ - 3 PUFA during pregnancy. The three bestknown benefits were optimal development of the fetal brain $(71.7 \%)$, decreased risk of hypertension/preeclampsia (43.4\%) and enhanced visual function of the child (39.6\%). Thirty-six (29.5\%) women did not know any dietary source of $n-3$ PUFA. The three best-known dietary sources were oily fish $(75.6 \%)$, rapeseed, walnut or linseed oil (45.4\%) and fish oil supplement (38.4\%).

Knowledge about $n-3$ PUFA was not influenced by the information source.

One hundred (82.0\%) pregnant women attached moderate or high importance to $n$-3 PUFA during pregnancy (Fig. 2). They would prefer receive information about $n-3$ PUFA from the gynaecologist-obstetrician $(53 \%)$, the general practitioner $(17 \%)$, the nutritionist 
(10\%), the midwife (3\%) or the media (1\%). Sixteen per cent did not have any preference.

The women who had already heard of or read information about $n$-3 PUFA were significantly more likely to perceive $n$-3 PUFA as important than women who did not (88.2 v. $71.4 \% ; P=0.02)$.

More than half $(52.5 \%)$ of all women reported changing their diet since they had become pregnant. Forty-two (34.4\%) declared paying particular attention to their consumption of $n$ - 3 PUFA, by including foods naturally rich in or enriched with $n-3$ PUFA in their diet ( $n 12)$ or by using $n$-3 LCPUFA supplements ( $n$ 23) or both ( $n 7)$. The proportion with favourable dietary practices regarding $n-3$ PUFA was significantly higher in women who had already heard of or read information about $n-3$ PUFA than in women who had not $(P=0.02)$, in women with good knowledge than in other women $(P=0.002)$, as well as in women who perceived $n$-3 PUFA as moderately or highly important than in women who perceived these nutrients as not or little important $(P=0 \cdot 006$; see Table 4$)$. Women who had received information about $n$-3 PUFA from their gynaecologist-obstetrician were also more likely to report favourable dietary practices than women who had not (66.7 v. 32.7\%; $P=0.0074)$.

Knowledge, attitude and dietary practices of pregnant women were not influenced by sociodemographic characteristics.

\section{Association between knowledge, attitude and dietary practices of the pregnant women and their omega-3 index}

After adjustment for sociodemographic features, the omega-3 index was significantly higher in pregnant women with favourable dietary practices regarding $n$-3 PUFA than in others $(\beta=0 \cdot 10$ (sE 0.05); $P=0 \cdot 04$ ).

Mediation analysis showed that dietary practices significantly mediated the relationship between knowledge and omega-3 index. The indirect effect was 0.323 (95\% CI 0.011, $0 \cdot 722$ ) and the mediation was complete. Dietary practices were not a mediator of the relationship between attitude and omega-3 index (indirect effect $=0 \cdot 174$ (95\% CI $-0 \cdot 141$, 0.549)). Moreover, there was no significant direct effect of attitude on the omega- 3 index. More results regarding the fatty acid profile of the participants can be found elsewhere ${ }^{(34)}$.

\section{Knowledge, attitude and clinical practices of gynaecologists-obstetricians}

While twenty-six (38.8\%) of the sixty-seven health-care professionals claimed that a suboptimal $n-3$ PUFA status is common in pregnant women, forty-one (61.2\%) reported this situation as non-existent or occasional. Fifty-four (81.8\%) recognized that guidelines on $n-3$ PUFA for pregnant women are needed. Health-care providers for whom low $n$ - 3 PUFA status is common were more likely to ask for guidelines than those for whom this situation is non-existent or occasional $(96.2 v .72 .5 \% ; P=0.02)$. Fiftythree $(79.1 \%)$ of the sixty-seven participants reported that their training was insufficient to inform their pregnant patients about $n$-3 PUFA. Forty-one (61.2\%) would be rather or very interested in receiving information.

Twenty (29.9\%) gynaecologists-obstetricians declared attaching moderate or high importance to $n-3$ PUFA during pregnancy (Fig. 2). This proportion was significantly higher in professionals for whom a suboptimal $n-3$ PUFA status is common in pregnant women than in those for whom this situation is non-existent or occasional $(53.9 \mathrm{v}$.

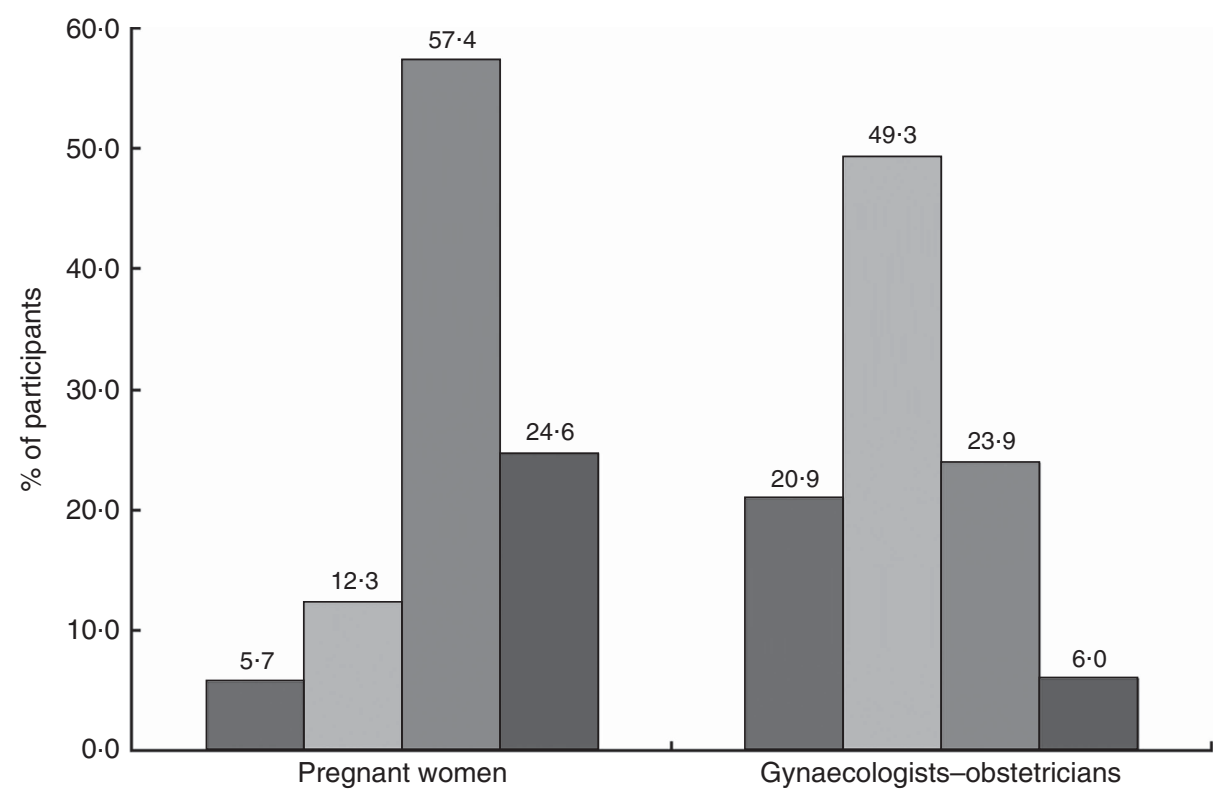

Fig. 2 Importance of $n$-3 PUFA during pregnancy ( $\square$, no importance; $\square$, little importance; $\square$, moderate importance; $\square$, high importance) according to pregnant women (n 122) and gynaecologists-obstetricians ( $n$ 67) sampled in Belgium, FebruaryAugust 2016 
Table 4 Number and proportion of pregnant women $(n$ 122) with favourable dietary practices regarding $n$-3 PUFA intake according to knowledge and attitude, Belgium, February-August 2016

\begin{tabular}{|c|c|c|c|c|c|}
\hline \multirow[b]{3}{*}{ Variable } & \multicolumn{4}{|c|}{ Favourable dietary practices } & \multirow[b]{3}{*}{$P$ value } \\
\hline & \multicolumn{2}{|c|}{ No } & \multicolumn{2}{|c|}{ Yes } & \\
\hline & $n$ & $\%$ & $n$ & $\%$ & \\
\hline Familiar with $n-3$ PUFA* & & & & & 0.02 \\
\hline Yes & 44 & 57.9 & 32 & $42 \cdot 1$ & \\
\hline No & 36 & $78 \cdot 3$ & 10 & $21 \cdot 7$ & \\
\hline Good knowledge about $n$-3 PUFA & & & & & 0.002 \\
\hline Yes & 20 & 43.5 & 26 & 56.5 & \\
\hline No & 23 & $79 \cdot 3$ & 6 & 20.7 & \\
\hline Attitude & & & & & 0.006 \\
\hline$n$-3 PUFA perceived as moderately or highly important & 60 & $60 \cdot 0$ & 40 & $40 \cdot 0$ & \\
\hline$n-3$ PUFA perceived as not or little important & 20 & $90 \cdot 9$ & 2 & 9.09 & \\
\hline
\end{tabular}

${ }^{*}$ Women familiar with $=$ women who have already heard of or read about $n-3$ PUFA.

$14.6 \% ; P=0.0006)$. Attitude did not differ according to demographic characteristics (sex, workplace and professional experience). More than half $(58 \cdot 2 \%)$ of the sixtyseven participants declared having one or several clinical practices regarding $n-3$ PUFA during pregnancy. Thirtyeight (56.7\%) claimed to give information about $n-3$ PUFA to their pregnant patients. Of the professionals, $53.7 \%$ reported implementing preventive actions when low $n-3$ PUFA levels were suspected: dietary advice for $18.2 \%$ of them, supplementation for $57.6 \%$ or the two combined strategies for $24.2 \%$. One gynaecologist-obstetrician systematically determined biomarkers of $n-3$ PUFA status in her patients, and six did it depending on the women's diet. The results are summarized in Table 5 .

The professionals' practice did not differ according to demographic characteristics or estimated frequency of suboptimal $n$-3 PUFA status. Gynaecologists-obstetricians who attached moderate or high importance to $n-3$ PUFA during pregnancy were more likely to have nutritional care practices than those who saw them as little or not important $(90.0$ v. $55.3 \% ; P=0.006)$. For one-third of obstetricians (33.3\%), a routine supplementation should be set up to ensure an adequate $n-3$ PUFA status in pregnant women. The reasons given included a healthy pregnancy and the optimal development of the fetus, or a diet low in $n$-3 PUFA. For $47 \%$, this strategy is not relevant for the following reasons: there is not enough evidence yet, nutritional support should be provided on a case-bycase basis, priority must be given to the $n-3$ PUFA from foods, or supplements are too expensive. The remaining $19.7 \%$ declared they could not respond due to a lack of knowledge. Health-care providers for whom a suboptimal $n-3$ PUFA status is common were more likely to be in favour of supplements $(59 \cdot 1 v \cdot 29 \cdot 0 \% ; P=0.03)$ than those for whom this situation is non-existent or occasional. The same trend was observed with health-care providers who attached moderate or high importance to $n$-3 PUFA during pregnancy (66.7 v. 28.6\%; $P=0.008)$.
Table 5 Detailed clinical practices of gynaecologists-obstetricians ( $n$ 67) regarding $n$-3 PUFA during pregnancy, Belgium, FebruaryMay 2016

\begin{tabular}{lrr}
\hline Variable & $n$ & $\%$ \\
\hline Information about $n$-3 PUFA given to pregnant & patients & \\
$\quad$ Never & 29 & 43.3 \\
Sometimes & 22 & 32.8 \\
Often & 12 & 17.9 \\
Always & 4 & $6 \cdot 0$ \\
Implementation of preventive actions & 31 & 46.3 \\
No & 36 & $53 \cdot 7$ \\
Yes & & \\
Implemented preventive actions & 6 & 18.2 \\
Dietary advice & 19 & $57 \cdot 6$ \\
Supplementation & 8 & 24.2 \\
Dietary advice and supplementation & \\
Determination of women's $n$-3 PUFA biomarkers & \\
$\quad$ Never & 60 & 89.6 \\
On the basis of women's diet & 6 & 9.0 \\
Systematically & 1 & 1.5 \\
\hline
\end{tabular}

\section{Discussion}

Over the past years, $n$-3 PUFA and pregnancy has been a topic of much research interest and controversy. While there is a lot of evidence supporting a beneficial role of $n-3$ LCPUFA in multiple maternal and child health outcomes, the typical Western diet has been found to be deficient in $n-3$ PUFA, with a relatively ratio of high $n-6$ PUFA to $n$ - 3 PUFA ${ }^{(39,40)}$. Pregnant women do not achieve the recommended intake of at least $200 \mathrm{mg}$ of DHA daily ${ }^{(41)}$. Although there are two major ways for increasing $n-3$ LCPUFA intake, there is no clear message on the best strategy to adopt. On the one hand, fish consumption may increase the maternal and fetal exposure to contaminants such as methylmercury, dioxins and polychlorinated biphenyls. Advice on fish consumption has consequently been found to be confusing and conflicting ${ }^{(27)}$. On the other hand, trials on fish or algal oil supplementation in 
pregnancy have shown some benefits for both mother and child; however, the results are still mixed and inconclusive $^{(42,43)}$. The discussion is definitely still ongoing, particularly in the light of recent scientific papers reporting no cardiovascular benefits from $n-3$ PUFA supplementation $^{(44-46)}$.

Several conditions are reminiscent of vitamin D: large media coverage, early and non-definitive research on health benefits, and the absence of consensual guidelines for the management of nutrient insufficiency. These have been shown to create a situation wherein both patients and health-care providers experience uncertainty ${ }^{(47,48)}$. In this context, the present study aimed at exploring knowledge, attitudes and practices of both pregnant women and gynaecologists-obstetricians regarding $n-3$ PUFA during pregnancy.

Our findings clearly highlighted a lack of knowledge among pregnant women. Only 38\% (forty-six out of 122) gave one or several correct facts about $n$-3 PUFA without prompt. Television, the Internet, books and magazines were reported to be the most common source of information. Only one-third of the pregnant women reported an increase in their $n$-3 PUFA intake, by including foods naturally rich in or enriched with $n-3$ PUFA in their diet and/or by using $n$-3 LCPUFA supplements. A positive association was observed between knowledge and attitude, and favourable dietary behaviours. This finding is consistent with others who found that awareness and nutrition knowledge have the potential to contribute to improving dietary behaviours ${ }^{(33,49)}$, even if a cause-andeffect relationship cannot be determined here. Statistical analyses were taken one step further; we investigated the relationship between self-declared dietary practices and an objective measure of $n$-3 PUFA status. The results confirmed that women with favourable dietary practices regarding n-3 PUFA were more likely to have higher omega-3 index. Furthermore, testing dietary practices as mediator revealed its role in the knowledge-omega-3 index relationship.

The current study revealed that only a limited number of pregnant women (twenty-two participants out of 122, $18 \%$ ) had already heard about $n-3$ PUFA during preconception or antenatal care. Australian pregnant women were not more privileged, since only $23 \%$ reported having received information ${ }^{(29)}$. From the limited number of studies, it is a major concern that the benefits of $n-3$ LCPUFA are being communicated to no more than one-quarter of pregnant women. Consistent with this finding, pregnant women were found to be relatively unaware of the health benefits of $n-3$ PUFA for both mother and fetus.

Pregnant women show a great interest in nutrition during their pregnancy ${ }^{(50,51)}$ and antenatal care provides a real opportunity for discussion around these topics. The participants in the present survey reported gynaecologists-obstetricians as a preferred channel of receiving information about $n-3$ PUFA. This source was associated with better knowledge related to $n$-3 PUFA and pregnancy, as well as with favourable dietary behaviours. This confirms the key role played by health-care providers in the education of their patients ${ }^{(52,53)}$. Assessment of the knowledge, attitude and practices of gynaecologistsobstetricians was seen as a prerequisite to improve $n-3$ PUFA intake in pregnant women.

Despite the well-documented roles of $n$-3 PUFA in pregnancy, related health-care professionals' awareness (knowledge and attitude) was rather poor. The current study highlighted the fact that gynaecologists-obstetricians underestimated the extent of suboptimal $n$ - 3 PUFA status in pregnant women. While two-thirds of health-care providers reported that this situation is non-existent or occasional, the determination of maternal erythrocyte $n-3$ PUFA levels revealed that almost all pregnant women (92\%) exhibited an IOM $3<8 \%{ }^{(34)}$. An IOM $3 \geq 8 \%$ was suggested as optimal for cardiovascular protection ${ }^{(36,54)}$. Precise levels of $n-3$ LCPUFA for a healthy pregnancy are unknown; however, some studies have already observed an association of omega-3 index with the risk of postpartum depression ${ }^{(55)}$ and with low birth weight and preterm delivery ${ }^{(56,57)}$. While pregnant women placed high importance on $n-3$ PUFA during pregnancy, such an attitude was observed in only one-third of gynaecologistsobstetricians. While a lack of time/survey burden is often the main reason for not responding to a survey ${ }^{(58,59)}$, the low participation rate could also reflect the lack of interest professionals have for the topic.

It is worth noting that $82 \%$ of the professionals showed interest in receiving guidelines on $n-3$ PUFA in pregnant women. This result questions the availability and accessibility of information materials and guidelines for gynaecologists-obstetricians. Very few resources about $n-3$ PUFA, or even fish consumption, exist in Wallonia, Belgium. Seventy-nine per cent of the professionals reported that their training regarding $n-3$ PUFA was insufficient. The present study sheds light on a lack of adequate physician training in nutrition; a worrying situation if we consider that early-life nutrition has long-term implications on the offspring's future health ${ }^{(60)}$. The uncertainty situation (as reported above), coupled with limited health professional training, could represent key barriers for gynaecologistsobstetricians to start advising their pregnant patients about the importance of $n$ - 3 LCPUFA.

Despite weak awareness regarding the importance of $n$ 3 PUFA during pregnancy, a little more than half of the gynaecologists-obstetricians reported taking preventive actions with pregnant patients with low $n$-3 PUFA intake. The most common strategy was $n-3$ LCPUFA supplementation. This finding can be surprising considering the conflicting scientific information. Unfortunately, no information on the recommended type of supplements and dose were collected in the current study. For one-third of the gynaecologists-obstetricians, a routine supplementation should even be implemented to ensure an adequate 
n-3 PUFA status in pregnant women. While supplementation can be a relatively simple way of increasing $n-3$ LCPUFA, some studies have found a significant variation in the quality of supplements ${ }^{(61)}$. Even if no serious deleterious effects were observed in pregnant women taking prenatal marine oil supplements ${ }^{(62)}$, benefits for both mother and child should be investigated further ${ }^{(43)}$.

Pregnant women are a priority public health target group. They should benefit from the promotion of the importance of $n$-3 LCPUFA consumption early in pregnancy (and ideally in preconception care) for optimal pregnancy outcomes. As specialists at the front line of antenatal care, gynaecologists-obstetricians could play multiple roles regarding $n$ - 3 LCPUFA. They could provide information, identify deficient diet, assess biomarkers of nutritional status and encourage behaviour changes. Increasing education of health-care providers about $n-3$ LCPUFA during pregnancy is clearly warranted. It is encouraging to note that two-thirds of the professionals expressed a desire for further information in the present study. Besides health-care providers' intervention, communication materials exist and could have the potential to assist in increased consumption of $n-3$ PUFA by pregnant women $^{(33)}$. Further research is needed to identify the most effective strategy concerning how antenatal care providers could promote all essential nutrients in pregnancy and enhance nutritional care provided to pregnant women.

Some limitations of the current study have to be noted. First, pregnant women and gynaecologists-obstetricians who agreed to participate in the survey may have a greater interest in the subject matter and therefore better knowledge, attitudes and practices related to $n-3$ PUFA. As previously discussed $^{(34)}$, pregnant women from the CHR Liège Hospital have some different sociodemographic features in comparison with the Walloon population. Considering knowledge, attitude and dietary practices of pregnant women were not influenced by sociodemographic characteristics, we could be confident in our conclusions. As the study was restricted to gynaecologistsobstetricians, we have no data on knowledge, attitude and clinical practices of other health-care providers involved in antenatal care such as midwives. Finally, as the survey conducted among gynaecologists-obstetricians was anonymous, no direct link could be established between the pregnant women and their specialist (e.g. specialist advice and behaviours of the patient). However, the number of specialists included would have been very limited since the women were from the same hospital.

\section{Conclusions}

In conclusion, the present study evidenced a need for pregnant women to receive adequate nutrition education and effective communication about n-3 PUFA. n-3 LCPUFA consumption during pregnancy is important.
Gynaecologists-obstetricians play an important role in the education of their patients and could discuss this issue during routine prenatal care, preferably during the first trimester. Evidence-based guidelines, refreshment training and communication tools could improve the professionals' awareness and clinical practices regarding $n$-3 LCPUFA, contributing to the health of mothers and their children. The important results of the present study should stimulate researchers to conduct more comprehensive research that reflects the scale of this major health problem in Belgium and elsewhere.

\section{Acknowledgements}

Acknowledgements: The authors would like to thank Elisabetha Prato and Marie Timmermans from the CHR Liège Hospital for their assistance in the data collection. They are also grateful to Professor Adelin Albert from the University of Liège for the critical revision of the manuscript. Financial support: This research was funded by the medical analysis laboratory Roman Païs (Belgium) (http://www.rplab.be); Metagenics Europe (https://www.metagenics.eu); and the University of Liège (Belgium) (https://www.uliege.be). The funders had no role in the study design, data collection and analysis, decision to publish, or preparation of the manuscript. Conflict of interest: None declared. Authorship: M.G. and V.C. are joint senior authors. A.H. was involved in the research design and the coordination of the survey, performed the statistical analyses and drafted the manuscript. F.B. conducted the data collection under the supervision of M.N. and S.D. M.N. and S.D. were also involved in the research design. A.-F.D. and N.D. took part in the statistical analyses and the critical discussion of the results. M.N. contributed to the critical revision and intellectual content of the manuscript. M.G. and V.C. as the promoters of the study supervised the entire work and finalized the manuscript. All authors read and approved the final version. Ethics of human subject participation: This study was conducted according to the guidelines laid down in the Declaration of Helsinki and all procedures involving human subjects were approved by the Ethics Committee of the CHR Citadelle Hospital of Liège, Belgium (B412201526650). Written informed consent was obtained from all pregnant women.

\section{References}

1. Connor WE (2000) Importance of $n-3$ fatty acids in health and disease. Am J Clin Nutr 71, 1 Suppl., 171S-175S.

2. Calder PC (2015) Functional roles of fatty acids and their effects on human health. JPEN J Parenter Enter Nutr 39, 1 Suppl., 18S-32S.

3. Uauy R, Mena P \& Rojas C (2000) Essential fatty acids in early life: structural and functional role. Proc Nutr Soc 59, 3-15.

4. Lauritzen L, Hansen HS, Jørgensen MH et al. (2001) The essentiality of long chain $n-3$ fatty acids in relation to 
development and function of the brain and retina. Prog Lipid Res 40, 1-94.

5. Clandinin MT, Chappell JE, Leong S et al. (1980) Intrauterine fatty acid accretion rates in human brain: implications for fatty acid requirements. Early Hum Dev 4, 121-129.

6. Innis SM (2007) Fatty acids and early human development. Early Hum Dev 83, 761-766.

7. Shulkin M, Pimpin L, Bellinger D et al. (2018) n-3 Fatty acid supplementation in mothers, preterm infants, and term infants and childhood psychomotor and visual development: a systematic review and meta-analysis. J Nutr 148, 409-418.

8. Salvig JD \& Lamont RF (2011) Evidence regarding an effect of marine $n-3$ fatty acids on preterm birth: a systematic review and meta-analysis. Acta Obstet Gynecol Scand 90 , 825-838.

9. Burchakov D, Kuznetsova I \& Uspenskaya Y (2017) Omega-3 long-chain polyunsaturated fatty acids and preeclampsia: trials say 'no,' but is it the final word? Nutrients 9 , 1364.

10. Lin P-Y, Chang C-H, Chong MF-F et al. (2017) Polyunsaturated fatty acids in perinatal depression: a systematic review and meta-analysis. Biol Psychiatry 82, 560-569.

11. Best KP, Gold M, Kennedy D et al. (2016) Omega-3 longchain PUFA intake during pregnancy and allergic disease outcomes in the offspring: a systematic review and metaanalysis of observational studies and randomized controlled trials. Am J Clin Nutr 103, 128-143.

12. Hanebutt FL, Demmelmair H, Schiessl B et al. (2008) Longchain polyunsaturated fatty acid (LC-PUFA) transfer across the placenta. Clin Nutr 27, 685-693.

13. Anon. (2009) Fats and fatty acids in human nutrition. Proceedings of the Joint FAO/WHO Expert Consultation. November 10-14, 2008. Geneva, Switzerland. Ann Nutr Metab 55, 5-300.

14. Agence nationale de sécurité sanitaire de l'alimentation, de l'environnement et du travail (2011) Actualisation des apports nutritionnels conseillés pour les acides gras - Rapport d'expertise collective. Maisons-Alfort: ANSES.

15. EFSA Panel on Dietetic Products Nutrition and Allergies (2012) Scientific Opinion on the Tolerable Upper Intake Level of eicosapentaenoic acid (EPA), docosahexaenoic acid (DHA) and docosapentaenoic acid (DPA). EFSA J 10, 2815.

16. de Groot RHM, Hornstra G, van Houwelingen AC et al. (2004) Effect of alpha-linolenic acid supplementation during pregnancy on maternal and neonatal polyunsaturated fatty acid status and pregnancy outcome. Am J Clin Nutr 79, 251-260.

17. Bernard JY, De Agostini M, Forhan A et al. (2013) The dietary n6:n3 fatty acid ratio during pregnancy is inversely associated with child neurodevelopment in the EDEN mother-child cohort. J Nutr 143, 1481-1488.

18. Olsen SF, Hansen HS, Sandström B et al. (1995) Erythrocyte levels compared with reported dietary intake of marine $n-3$ fatty acids in pregnant women. Br J Nutr 73, 387-395.

19. Sioen I, van Lieshout L, Eilander A et al. (2017) Systematic review on $n-3$ and $n-6$ polyunsaturated fatty acid intake in European countries in light of the current recommendations - focus on specific population groups. Ann Nutr Metab 70 , $39-50$.

20. Food and Agriculture Organization of the United Nations (2005) Food-based dietary guidelines - Belgium. http:// www.fao.org/nutrition/education/food-based-dietary-guide lines/regions/countries/belgium/en/ (accessed November 2018).

21. Al MD, van Houwelingen AC, Kester AD et al. (1995) Maternal essential fatty acid patterns during normal pregnancy and their relationship to the neonatal essential fatty acid status. Br J Nutr 74, 55-68.
22. Otto SJ, Houwelingen AC, Antal M et al. (1997) Maternal and neonatal essential fatty acid status in phospholipids: an international comparative study. Eur J Clin Nutr 51, 232-242.

23. Hornstra G, Al MD, van Houwelingen AC et al. (1995) Essential fatty acids in pregnancy and early human development. Eur J Obstet Gynecol Reprod Biol 61, 57-62.

24. Committee on the Toxicological Effects of Methylmercury, National Research Council (2000) Toxicological Effects of Methylmercury. Washington, DC: National Academies Press.

25. Oken E, Kleinman KP, Berland WE et al. (2003) Decline in fish consumption among pregnant women after a national mercury advisory. Obstet Gynecol 102, 346-351.

26. Taylor CM, Emmett PM, Emond AM et al. (2018) A review of guidance on fish consumption in pregnancy: is it fit for purpose? Public Health Nutr 21, 2149-2159.

27. Forbes L, Graham J, Berglund C et al. (2018) Dietary change during pregnancy and women's reasons for change. Nutrients 10, 1032

28. Verbeke W, Sioen I, Pieniak Z et al. (2005) Consumer perception versus scientific evidence about health benefits and safety risks from fish consumption. Public Health Nutr $\mathbf{8}$, 422-429.

29. Sinikovic DS, Yeatman HR, Cameron D et al. (2009) Women's awareness of the importance of long-chain omega-3 polyunsaturated fatty acid consumption during pregnancy: knowledge of risks, benefits and information accessibility. Public Health Nutr 12, 562-569.

30. Aaronson LS, Mural CM \& Pfoutz SK (1988) Seeking information: where do pregnant women go? Health Educ Q $\mathbf{1 5}$, $335-345$

31. May L, Suminski R, Berry A et al. (2014) Diet and pregnancy: health-care providers and patient behaviors. J Perinat Educ 23, 50-56.

32. Bloomingdale A, Guthrie LB, Price S et al. (2010) A qualitative study of fish consumption during pregnancy. Am J Clin Nutr 92, 1234-1240.

33. Emmett R, Akkersdyk S, Yeatman H et al. (2013) Expanding awareness of docosahexaenoic acid during pregnancy. Nutrients 5, 1098-1109.

34. Hoge A, Bernardy F, Donneau A-F et al. (2018) Low omega3 index values and monounsaturated fatty acid levels in early pregnancy: an analysis of maternal erythrocytes fatty acids. Lipids Health Dis 17, 63.

35. Harris WS \& Von Schacky C (2004) The Omega-3 Index: a new risk factor for death from coronary heart disease? Prev Med 39, 212-220.

36. Harris WS (2007) Omega-3 fatty acids and cardiovascular disease: a case for omega-3 index as a new risk factor. Pharmacol Res 55, 217-223.

37. Harris WS, Del Gobbo L \& Tintle NL (2017) The Omega-3 Index and relative risk for coronary heart disease mortality: estimation from 10 cohort studies. Atherosclerosis 262, $51-54$.

38. Valeri L \& Vanderweele TJ (2013) Mediation analysis allowing for exposure-mediator interactions and causal interpretation: theoretical assumptions and implementation with SAS and SPSS macros. Psychol Methods 18, 137-150.

39. Stark KD, Van Elswyk ME, Higgins MR et al. (2016) Global survey of the omega-3 fatty acids, docosahexaenoic acid and eicosapentaenoic acid in the blood stream of healthy adults. Prog Lipid Res 63, 132-152.

40. Micha R, Khatibzadeh S, Shi P et al. (2014) Global, regional, and national consumption levels of dietary fats and oils in 1990 and 2010: a systematic analysis including 266 countryspecific nutrition surveys. BMJ 348, g2272.

41. Koletzko B, Lien E, Agostoni C et al. (2008) The roles of long-chain polyunsaturated fatty acids in pregnancy, 
lactation and infancy: review of current knowledge and consensus recommendations. J Perinat Med 36, 5-14.

42. Imhoff-Kunsch B (2011) e-Library of Evidence for Nutrition Actions (eLENA) I Marine oil supplementation in pregnancy and maternal and neonatal health outcomes. http://www. who.int/elena/titles/commentary/fish_oil_pregnancy/en/ (accessed January 2018).

43. Rogers LK, Valentine CJ \& Keim SA (2013) DHA supplementation: current implications in pregnancy and childhood. Pharmacol Res 70, 13-19.

44. Elagizi A, Lavie CJ, Marshall K et al. (2018) Omega-3 polyunsaturated fatty acids and cardiovascular health: a comprehensive review. Prog Cardiovasc Dis 61, 76-85.

45. Aung T, Halsey J, Kromhout D et al. (2018) Associations of omega-3 fatty acid supplement use with cardiovascular disease risks. JAMA Cardiol 3, 225-234.

46. Abdelhamid AS, Brown TJ, Brainard JS et al. (2018) Omega3 fatty acids for the primary and secondary prevention of cardiovascular disease. Cochrane Database Syst Rev 7, CD003177.

47. Bennett K, Frisby BN, Young LE et al. (2014) Vitamin D: an examination of physician and patient management of health and uncertainty. Qual Health Res 24, 375-386.

48. Bianchi CM, Huneau J-F, Le Goff G et al. (2016) Concerns, attitudes, beliefs and information seeking practices with respect to nutrition-related issues: a qualitative study in French pregnant women. BMC Pregnancy Childbirth 16, 306.

49. Wardle J, Parmenter K \& Waller J (2000) Nutrition knowledge and food intake. Appetite 34, 269-275.

50. Szwajcer EM, Hiddink GJ, Koelen MA et al. (2005) Nutritionrelated information-seeking behaviours before and throughout the course of pregnancy: consequences for nutrition communication. Eur J Clin Nutr 59, Suppl. 1, S57-S65.

51. Bookari K, Yeatman H \& Williamson M (2017) Informing nutrition care in the antenatal period: pregnant women's experiences and need for support. Biomed Res Int $\mathbf{2 0 1 7}$ 4856527.

52. Kreuter MW, Chheda SG \& Bull FC (2000) How does physician advice influence patient behavior? Evidence for a priming effect. Arch Fam Med 9, 426-433.

53. Quader ZS, Cogswell ME, Fang J et al. (2017) Changes in primary healthcare providers' attitudes and counseling behaviors related to dietary sodium reduction, DocStyles 2010 and 2015. PLoS One 12, e0177693.

54. von Schacky C (2011) The Omega-3 Index as a risk factor for cardiovascular diseases. Prostaglandins Other Lipid Mediat 96, 94-98.

55. Markhus MW, Skotheim S, Graff IE et al. (2013) Low omega3 index in pregnancy is a possible biological risk factor for postpartum depression. PLoS One 8, e67617.

56. Ogundipe E, Johnson MR, Wang Y et al. (2016) Periconception maternal lipid profiles predict pregnancy outcomes. Prostaglandins Leukot Essent Fatty Acids 114, $35-43$.

57. Klebanoff MA, Harper M, Lai Y et al. (2011) Fish consumption, erythrocyte fatty acids, and preterm birth. Obstet Gynecol 117, 1071-1077.

58. Cook DA, Wittich CM, Daniels WL et al. (2016) Incentive and reminder strategies to improve response rate for internet-based physician surveys: a randomized experiment. J Med Internet Res 18, e244.

59. Dykema J, Jones NR, Piché T et al. (2013) Surveying clinicians by web. Eval Health Prof 36, 352-381.

60. Berti C, Cetin I, Agostoni C et al. (2016) Pregnancy and infants' outcome: nutritional and metabolic implications. Crit Rev Food Sci Nutr 56, 82-91.

61. Jordan RG (2010) Prenatal omega-3 fatty acids: review and recommendations. J Midwifery Womens Health $\mathbf{5 5}$, $520-528$

62. Freeman MP \& Sinha P (2007) Tolerability of omega-3 fatty acid supplements in perinatal women. Prostaglandins Leukot Essent Fatty Acids 77, 203-208. 